\section{Ultrasonographic findings of low-grade endometrial stromal sarcoma of the uterus with a focus on cystic degeneration}

\author{
Ga Eun Park', Sung Eun Rha', Soon Nam Oh' ${ }^{1}$, Ahwon Lee ${ }^{2}$, Keun Ho Lee ${ }^{3}$, Mee-Ran Kim³ \\ Departments of ${ }^{1}$ Radiology, ${ }^{2}$ Hospital Pathology, and ${ }^{3}$ Obstetrics and Gynecology, Seoul St. \\ Mary's Hospital, College of Medicine, The Catholic University of Korea, Seoul, Korea
}

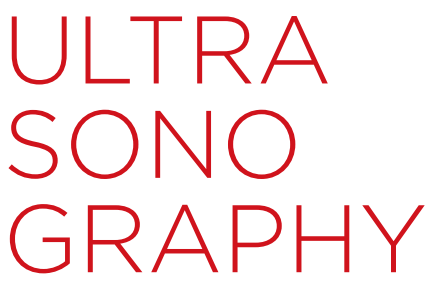

ORIGINAL ARTICLE

http://dx.doi.org/10.14366/usg. 15045 pISSN: 2288-5919 - eISSN: 2288-5943 Ultrasonography 2016;35:124-130

Received: August 10, 2015 Revised: September 22, 2015 Accepted: October 5, 2015

Correspondence to:

Sung Eun Rha, MD, Department of Radiology, Seoul St. Mary's Hospital, College of Medicine, The Catholic University of Korea, 222 Banpo-daero, Seocho-gu, Seoul 06591, Korea

Tel. $+82-2-2258-1430$

Fax. +82-2-599-6771

E-mail: serha@catholic.ac.kr

This is an Open Access article distributed under the terms of the Creative Commons Attribution NonCommercial License (http://creativecommons.org/ licenses/by-nc/3.0/) which permits unrestricted noncommercial use, distribution, and reproduction in any medium, provided the original work is properly cited.

Copyright (C) 2016 Korean Society of Ultrasound in Medicine (KSUM)

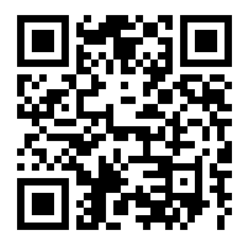

Low-grade endometrial stromal sarcoma (ESS) is a rare type of tumor of mesenchymal origin, accounting for $0.2 \%$ of all uterine malignancies, with an annual incidence of $1-2$ per million women. It is second most common purely mesenchymal tumor of the uterus, accounting for $15 \%-26 \%$ of uterine sarcomas [1].

In contrary to other uterine sarcomas, such as carcinosarcoma, leiomyosarcoma, and high-grade ESS, low-grade ESS generally involves a slowly growing malignancy with an indolent clinical course
How to cite this article:

Park GE, Rha SE, Oh SN, Lee A, Lee KH, grade endometrial stromal sarcoma of the uterus with a focus on cystic degeneration. Ultrasonography. 2016 Apr;35(2):124-130. Kim MR. Ultrasonographic findings of low- 
and late recurrence. Low-grade ESS patients tend to be younger than typical patients with other uterine sarcomas, with a mean age of 52 years [2]. The most common clinical presentation of low-grade ESS includes abnormal vaginal bleeding and pelvic or abdominal pain; consequently, low-grade ESS is often clinically difficult to differentiate from benign leiomyoma. However, the preoperative diagnosis of low-grade ESS is important because the current standard treatment for early-stage (stage I or II) low-grade ESS is hysterectomy and bilateral salpingo-oophorectomy for hormonally sensitive tumors, which is different from the treatment of the more common condition of benign leiomyoma $[3,4]$. The preoperative differential diagnosis of low-grade ESS is difficult due to its rarity and the lack of pathognomonic imaging findings; therefore, most patients are preoperatively misdiagnosed with leiomyoma.

To the best of our knowledge, few reports have described the imaging findings associated with low-grade ESS, and previous studies were based on small numbers of patients due to the rarity of these tumors [5-10]. Ultrasound is an initial diagnostic tool for patients with abnormal vaginal bleeding or pelvic pain, as well as for the evaluation of uterine disease. Therefore, we examined the ultrasonographic findings of pathologically proven cases of lowgrade ESS in our institution and reviewed the literature on the imaging features of low-grade ESS.

\section{Materials and Methods}

Our Institutional Review Board approved this retrospective study and waived informed patient consent. We searched the medical database of our institute for patients who were treated between January 2007 and April 2014, and found 16 patients with pathologically confirmed endometrial stromal tumors (ESTs), including 11 cases of pathologically confirmed low-grade ESS, two cases of undifferentiated uterine sarcoma (UUS), and three cases of endometrial stromal nodules. The pathologic classification of ESTs was recently changed; therefore, the diagnoses of the identified cases were re-evaluated and confirmed by a single pathologist using the latest 2014 World Health Organization (WHO) classification [11]. One of the 11 patients with low-grade ESS did not undergo preoperative transvaginal sonography and was therefore excluded from the study. We ultimately included 10 patients with histopathologically proven low-grade ESS who underwent preoperative transvaginal sonography. One patient had both initial and 20-month follow-up ultrasonographic images before surgery. We retrospectively reviewed the medical records of each patient and extracted clinical, surgical, and pathological data. The patients ranged from 34 to 65 years of age (mean, 48 years). Eight patients were premenopausal and two patients were postmenopausal. No patient had a history of pelvic irradiation or tamoxifen administration for breast cancer; however, one patient had underlying systemic lupus erythematosus.

All patients were examined using transvaginal ultrasonography at our institution, using a range of machines, including the ProSound Alpha10 (Hitachi-Aloka, Tokyo, Japan), ProSound SSD3500SX (Hitachi-Aloka), Medison Accuvix XG (Samsung Medison, Seoul, Korea), and SonoAce X4 (Samsung Medison) ultrasound systems with an end-viewing endovaginal 5-9-MHz transducer. All ultrasound examinations were performed within 2 weeks prior to surgery.

Two radiologists retrospectively reviewed the ultrasonographic findings, coming to a consensus regarding the tumor location (purely intramural mass, intramural mass protruding into the endometrial cavity, or purely intracavitary mass), tumor size (maximal diameter of the tumor), tumor margin with the underlying myometrium (well-defined or ill-defined), echogenicity of solid portion of tumor compared to the normal myometrium (hyperechoic, isoechoic, or hypoechoic), the presence of intratumoral anechoic cysts, and the size and configuration of cysts (multilocular or unilocular).

Histological specimens were surgically obtained from all patients. One pathologist with 16 years of experience in gynecological oncology reviewed the microscopic examinations and immunohistochemical staining results of the ESTs and confirmed the diagnosis of low-grade ESS using the latest 2014 WHO classification [11,12]. Microscopically, the tumors consisted of cells resembling stromal cells of the proliferative-phase endometrium, showing infiltrative growth into the myometrium. We assessed the disease stage retrospectively for each patient using the 2009 International Federation of Gynecology and Obstetrics (FIGO) staging system for uterine sarcomas [13].

\section{Results}

\section{Clinical and Pathological Features}

The ten patients with low-grade ESS were presented with symptoms including menorrhagia $(n=4)$, abdominal pain $(n=2)$, and postmenopausal vaginal bleeding $(n=1)$. Three patients were scheduled for surgery due to the size increasement of a presumed leiomyoma during follow-up. The preoperative cancer antigen 125 (CA 125) levels of nine patients were within the normal range (0$35 \mathrm{IU} / \mathrm{mL}$ ), but one patient with a $9.2-\mathrm{cm}$ endometrial right ovary cyst had a CA 125 level of $135.05 \mathrm{IU} / \mathrm{mL}$. Preoperative endometrial biopsy was performed on two patients (cases 4 and 6) who presented with vaginal bleeding, and these patients were diagnosed with ESS before surgery. The preoperative ultrasonographic diagnosis for the other eight patients was leiomyoma. Preoperative magnetic resonance imaging was performed on seven patients, and the possibility of ESS was raised, leading to intraoperative frozen biopsy. 
Table 1. Ultrasonographic features of 10 cases confirmed as low-grade endometrial stromal sarcoma

\begin{tabular}{ccccccc}
\hline \multirow{2}{*}{ Case No. } & Age $(\mathrm{yr})$ & \multicolumn{5}{c}{ Tumor } \\
\cline { 3 - 6 } & & Size $(\mathrm{cm})$ & Location & Margin & Echogenicity & Shape \\
\hline 1 & 51 & 6.2 & IM & Well-defined & Hyperechoic & Solid mass with multiseptated cystic area \\
2 & 34 & 7.6 & IM+EM & Well-defined & Isoechoic & Solid mass with multiseptated cystic area \\
4 & 47 & 6.3 & IM+EM & Well-defined & Hyperechoic & Solid mass with multiple small cysts \\
5 & 65 & 4.3 & IM+EM & III-defined & Hyperechoic & Solid mass with multiple small cysts \\
6 & 46 & 7 & IM+EM & Well-defined & Isoechoic & Solid mass with multiple small cysts \\
7 & 52 & 9.1 & IM+EM & III-defined & Isoechoic & Solid mass with unilocular cystic area \\
8 & 51 & 4.7 & IM & Well-defined & Isoechoic & Predominantly unilocular cystic mass \\
9 & 50 & 5.9 & IM & III-defined & Isoechoic & Diffuse infiltrative solid mass \\
10 & 41 & 7.5 & IM & III-defined & Hyperechoic & Diffuse infiltrative solid mass \\
\hline
\end{tabular}

${ }^{a}$ Echogenicity of solid tumor portion.

IM, intramural mass; IM+EM, intramural mass protruding into the endometrial cavity.

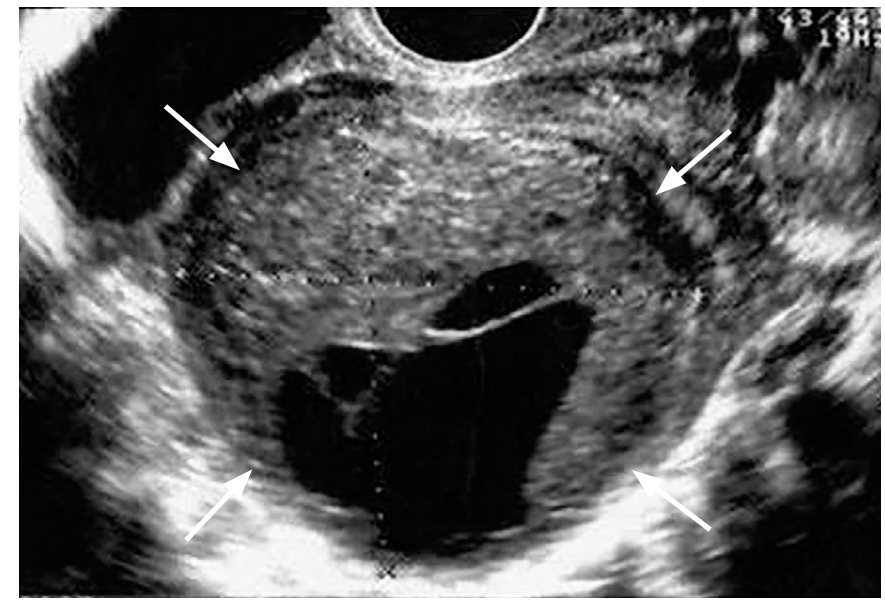

A

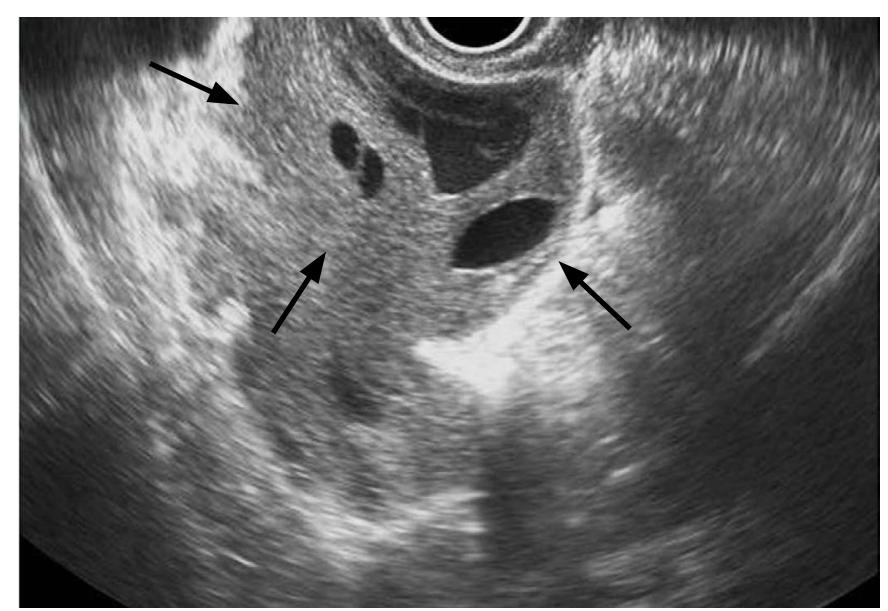

C

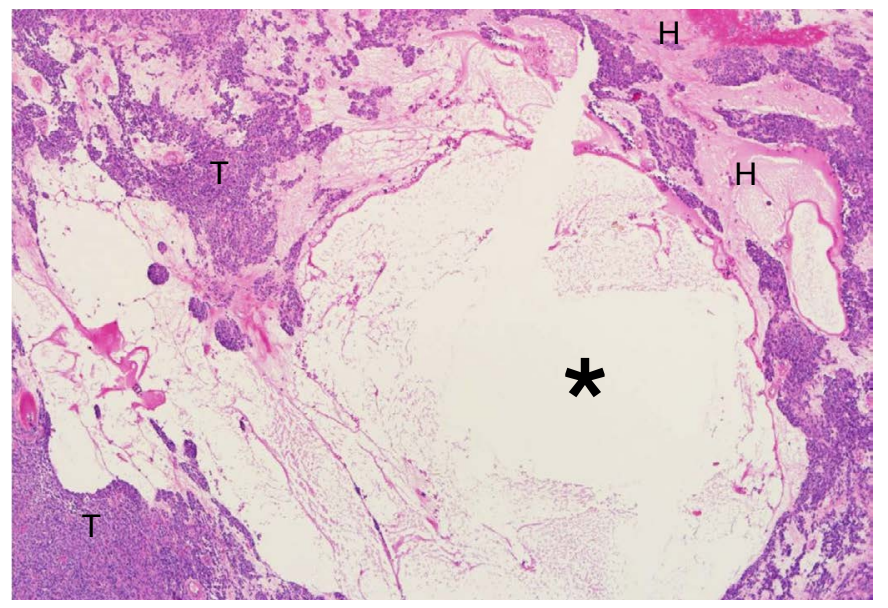

B

Fig. 1. A 34-year-old premenopausal woman with low-grade endometrial stromal sarcoma (case 2).

A. Transvaginal sonogram of the uterus shows a well-defined isoechoic mass (arrows) containing a multiseptated cystic portion. $B$. Photomicrograph of the resected tumor shows prominent cystic (asterisk) and hyaline $(\mathrm{H})$ degeneration, with sarcoma cells (T) infiltrating the myometrium $(H \& E, \times 40)$. C. Follow-up transvaginal sonogram 56 months after total hysterectomy shows a recurrent solid mass (arrows) containing multiple well-defined cystic portions. 
Ten patients eventually underwent total hysterectomy with bilateral salpingo-oophorectomy. Two patients (cases 2 and 9) initially underwent myomectomy, followed by secondary total hysterectomy with bilateral salpingo-oophorectomy after obtaining a pathologic diagnosis of low-grade ESS. In four patients, additional pelvic lymph node dissection or biopsy was performed if invasion or severe adhesion between the uterus and adjacent structures was suspected.

Nine patients had FIGO stage I tumors and one patient (case 6) had a stage IIA tumor displaying left adnexal involvement. One patient (case 8) underwent one cycle of chemotherapy after surgery due to suspected vascular invasion in the pathologic examination. The duration of postoperative follow-up ranged from 5 to 91 months (mean, 37.3 months). During the period of our study, one patient (case 2) experienced a recurrent tumor in the pelvic cavity 56 months after surgery.

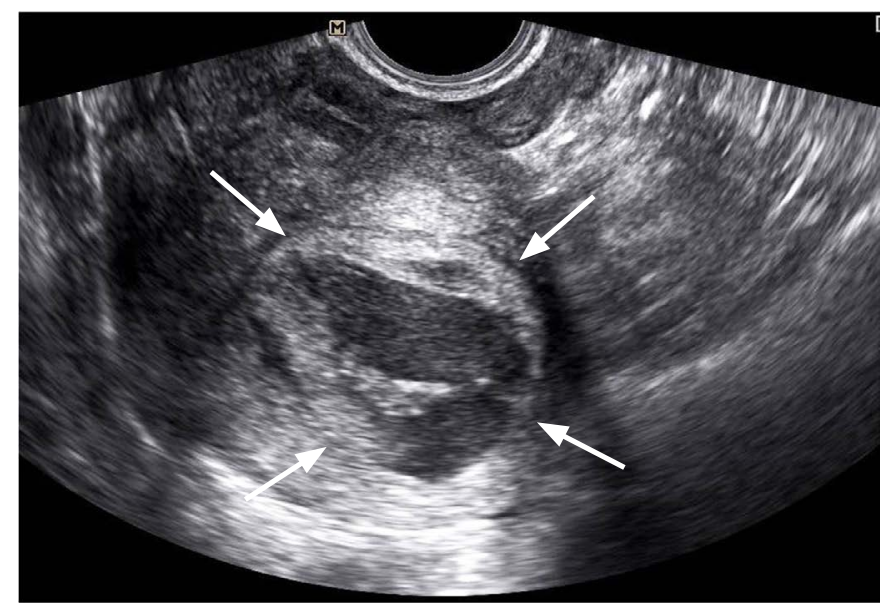

A

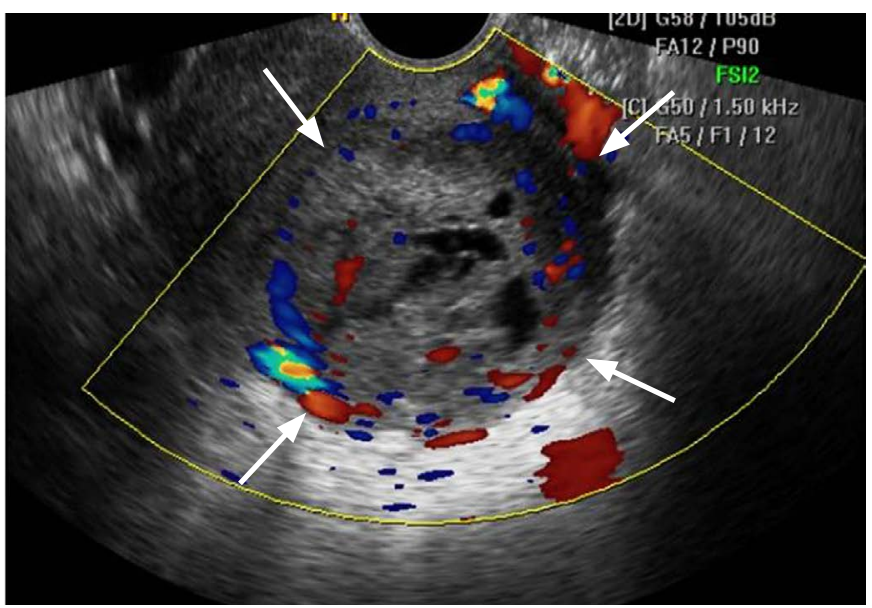

C

\section{Ultrasonographic Features}

Table 1 presents the ultrasonographic features of the 10 cases analyzed in this study. Low-grade ESS manifested as an intramural mass protruding into the endometrial cavity $(n=6)$ or as a purely intramural mass $(n=4)$. No exclusively intracavitary lesions were observed. The maximal diameters of the lesions ranged from 4 to $9.1 \mathrm{~cm}$ (mean, $6.2 \mathrm{~cm}$ ). The tumor margin with the underlying myometrium was smooth and well-defined in six patients and illdefined in four patients. The echogenicity of the solid portion of tumor was hyperechoic $(n=5)$ or isoechoic $(n=5)$ compared to the normal myometrium. Seven cases showed an internal well-defined anechoic cystic area. The size of the cystic lesions ranged from 1.3 to $4.5 \mathrm{~cm}$ (mean, $2.8 \mathrm{~cm}$ ). Five cases had a multiseptated cystic area (cases 1 and 2) (Fig. 1) or multiple clustered cystic areas (cases 3-5) (Fig. 2B), while two other cases showed a unilocular cystic area within the tumor (cases 6 and 7) (Fig. 3). We categorized lowgrade ESS into four patterns based on the ultrasonographic findings: a predominantly solid mass containing cystic areas $(n=6$, cases $1-6)$

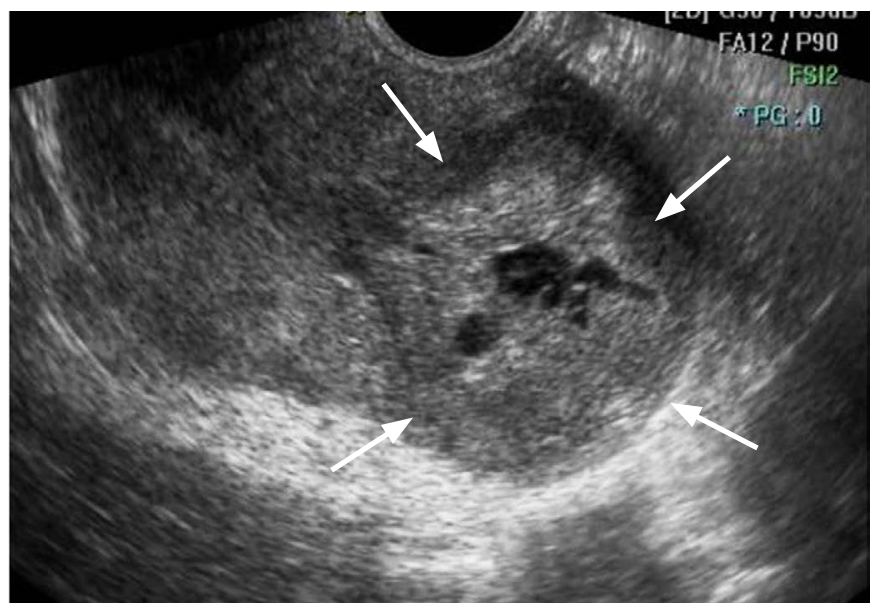

B

Fig. 2. A 47-year-old premenopausal woman with low-grade endometrial stromal sarcoma (case 3).

A. Initial transvaginal sonogram of the uterus shows a 4-cm welldefined intramural mass (arrows) protruding into the endometrial cavity. A large multiseptated cystic portion is visible within the solid tumor. This patient underwent follow-up under the impression of degenerated myoma. B. Follow-up transvaginal sonogram obtained 20 months after the initial transvaginal ultrasound shows a larger tumor with a decreased extent of internal cystic degeneration (arrows). C. Color Doppler image of the uterus at the same time as panel $B$ shows increased vascularity in the solid portion of the tumor (arrows). 


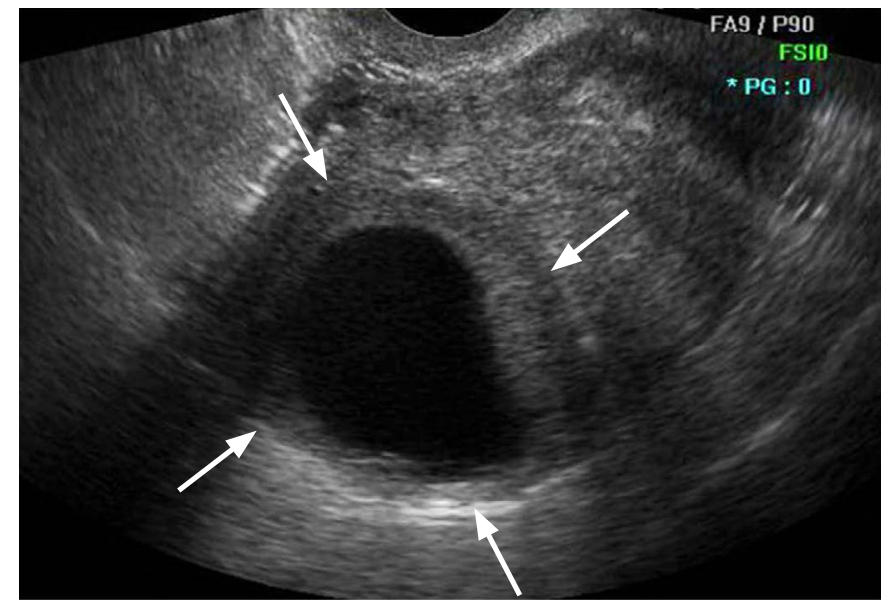

A

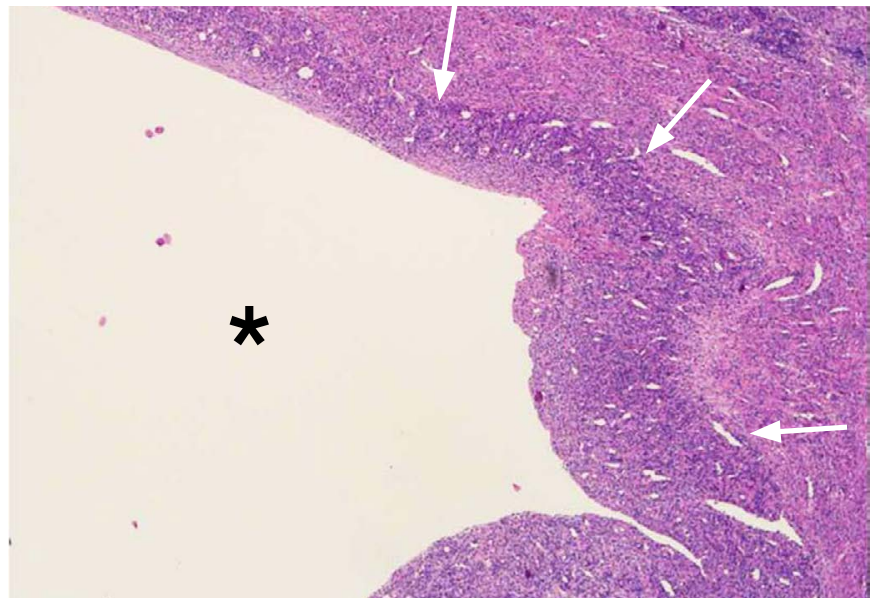

B

Fig. 3. A 51-year-old premenopausal woman with low-grade endometrial stromal sarcoma (case 7).

A. Transvaginal sonogram of the uterus shows a predominantly unilocular cystic mass with a peripheral isoechoic solid portion (arrows). B. Photomicrograph of the resected tumor shows a large unilocular cyst (asterisk) surrounded by sheets of infiltrating sarcoma cells (arrows) (H\&E, $\times 40)$.

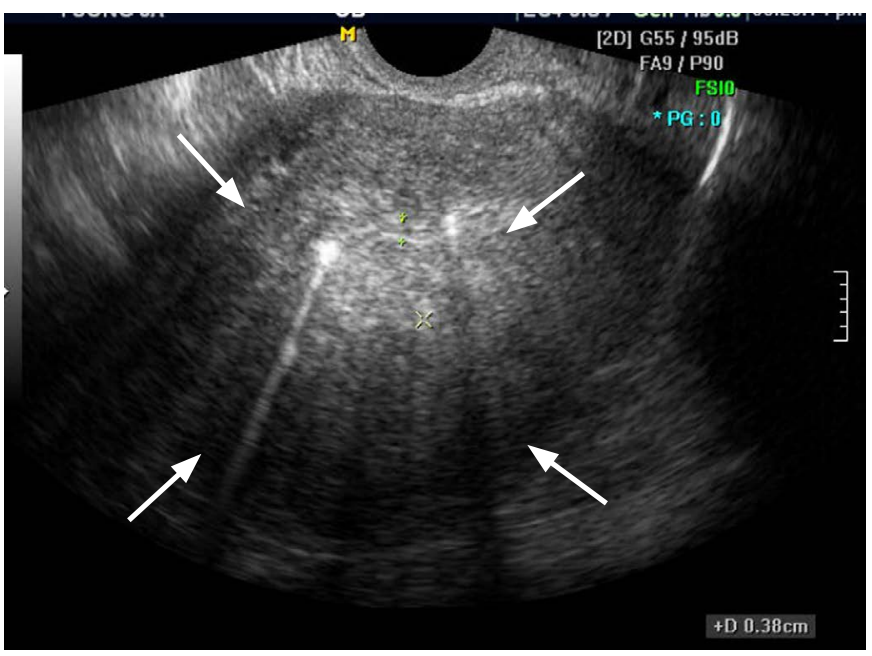

Fig. 4. A 50-year-old premenopausal woman with low-grade endometrial stromal sarcoma (case 8). Transvaginal ultrasound image of the uterus shows a diffusely enlarged uterus with an illdefined infiltrative intramural mass (arrows), mimicking diffuse adenomyosis.

(Figs. 1, 2), a predominantly unilocular cystic mass ( $n=1$, case 7 ) (Fig. 3), an ill-defined infiltrative solid mass mimicking adenomyosis $(n=2$, cases 8 and 9) (Fig. 4), and a well-defined solid mass ( $n=10$, case 10). With regard to other ultrasonographic findings, no patients had other pelvic peritoneal masses or ascites. In one patient (case 2), follow-up sonography 56 months after surgery revealed a large recurrent solid mass with several well-defined anechoic cystic areas in the pelvic cavity (Fig. 1C).

\section{Discussion}

The ultrasonographic features of low-grade ESS in our study were variable in terms of tumor location and shape, with the following findings: a mass protruding into the uterine cavity from the myometrium (60\%), a well-demarcated intramural mass $(20 \%)$, and an ill-defined intramural mass showing diffuse myometrial thickening $(20 \%)$. The margin of the mass was well-defined and smooth in six patients $(60 \%)$ and ill-defined in four patients (40\%). However, all cases were found to have infiltrative margins on microscopy.

In this study, seven patients (70\%) showed tumors with small to large internal cystic portions. Five cases had a multiseptated cystic appearance $(20 \%)$ or a multiple clustered small cystic appearance $(30 \%)$, and two cases $(20 \%)$ showed unilocular cystic features within the tumor. Interestingly, one case manifested as a $4.7-\mathrm{cm}$ intramural unilocular cystic mass with a thin peripheral solid wall. To the best of our knowledge, the imaging features of low-grade ESS with cystic degeneration have not been focused in the current literature. However, several sporadic case reports assessing the cystic appearance of low-grade ESS have described huge uterine masses $(8-15 \mathrm{~cm})$ with a multilocular cystic mass containing clear serous fluid [14-16]. We were able to find some reports describing large cystic degeneration or a multicystic appearance in low-grade ESS $[5,6,8,10]$, and a case report of low-grade ESS with multiple areas of small cystic degeneration [17]. From a pathologic perspective, one report stated that the cystic areas in cases of low-grade ESS resulted from the cystic dilatation of several endometrial glands that contained altered blood within the lumen [18]. However, 
no evidence of an endometrial gland component was found in the cystic lesions when we reviewed the pathologic slides of our patients; instead, we noted areas of cystic degeneration surrounded by endometrial stromal differentiated cells.

Leiomyoma is the most important differential diagnosis of lowgrade ESS based on clinical and imaging findings. Leiomyoma is more common, and a preoperative diagnosis of low-grade ESS therefore requires a high degree of suspicion towards an abnormal presentation, such as rapid growth or degenerative change. Although it is difficult to diagnose low-grade ESS based on ultrasonographic features, the high frequency of cystic degeneration that we observed might be a factor supporting the inclusion of low-grade ESS in the differential diagnosis of leiomyoma. Cystic degeneration is known to be one type of leiomyoma degeneration, with an incidence of 4\% [19]; however, seven out of the 10 cases in our study with low-grade ESS showed cystic degeneration. Although the incidence of cystic degeneration of low-grade ESS has not been reported, and our study contained a small number of cases, we think that cystic degeneration is not rare in low-grade ESS based on our case series and literature review.

Adenomyosis is another important differential diagnosis of lowgrade ESS presenting with diffuse myometrial thickening. In our opinion, the differential diagnosis of these diseases is almost impossible based on ultrasonographic features alone. However, one previous report concluded that a more nodular and coarse appearance of the myometrium may be suggestive of low-grade ESS instead of adenomyosis [8]. In our study, the masses showed a relatively echogenic and coarse appearance.

The classification of ESTs has been revised several times according to changes in the understanding of the biology and pathology of these rare tumors. ESS was initially divided into low-grade ESS and high-grade ESS based on mitotic activity per high-power field. However, mitotic activity was found to be an unreliable indicator of clinical behavior, and ESS was therefore reclassified into ESS (previously low-grade ESS) and undifferentiated endometrial sarcoma (previously high-grade ESS) in the 2003 WHO classification. Subsequently, a subset of ESS with a unique YWHAE-FAM22 gene rearrangement was discovered, and the high-grade ESS category was re-established as a subset of ESS with a prognosis intermediate between low-grade ESS and UUS. The latest 2014 WHO classification divided ESTs into four subtypes: endometrial stromal nodules, lowgrade ESS, high-grade ESS, and UUS [12]. Low-grade ESS is the most common EST and is an indolent malignant tumor with a good prognosis.

Low-grade ESS usually occurs at an earlier age than other uterine malignancies, and over $50 \%$ of low-grade ESS patients are premenopausal women. Patients commonly present with abnormal uterine bleeding, pelvic pain, and dysmenorrhea; however, as many as $25 \%$ of patients are asymptomatic [1-4]. In our study, the mean age of the patients was 48 years, and eight of the 10 patients were premenopausal. The main symptom of our patients was abnormal vaginal bleeding, which corresponds closely with previously published reports.

Macroscopically, low-grade ESS may be submucosal or intramural, usually with ill-defined borders and worm-like permeation within the myometrium and parametrium. There may be areas of cystic degeneration, hemorrhage, and necrosis within the tumor. Uncommonly, low-grade ESS may appear as a pure cystic mass [20]. Microscopically, low-grade ESS typically involves the endometrium and infiltrates the myometrium, often with lymphovascular invasion $[19,20]$.

Low-grade ESS is characterized by a prolonged disease-free interval and a 5 -year survival rate exceeding $90 \%$ for FIGO stage I and II tumors [11]. However, $45 \%$ of stage I patients experience late recurrences of metastasis that require long-term follow-up [21]. In our study, one recurrent tumor developed in the pelvic cavity 56 months after surgery. Follow-up imaging showed a large solid mass with multiple cystic degeneration, similar to the primary tumor. Previous case reports have described recurrent low-grade ESS presenting as a huge multiseptated cystic mass in the pelvic cavity [22], recurrent low-grade ESS unexpectedly found as a cystic mass in the right atrium [23], and cystic metastasis of low-grade ESS to the lungs [24].

Our study has some limitations. First, the study was based on retrospective consensus review and included only a small number of patients with low-grade ESS, which may not have accurately reflected the full spectrum of these tumors. However, low-grade ESS is a rare uterine malignancy, and our study is the largest extant study reviewing the ultrasonographic features of patients with lowgrade ESS according to the latest 2014 WHO classification. Second, Doppler sonography was not routinely obtained in every patient, so we could not review the vascular features of low-grade ESS. Third, a comparative analysis of the ultrasonographic findings between lowgrade ESS and other uterine tumors was not performed.

In conclusion, low-grade ESS shows variable imaging findings, with masses that can be well-defined or ill-defined, and involve diffuse myometrial thickening. The mass is usually located in the myometrium, but may protrude into the endometrial cavity. In addition, multiseptated cystic degeneration or multiple small areas of cystic degeneration are common, and a unilocular cystic appearance can also be an imaging finding of low-grade ESS. Therefore, lowgrade ESS should be included in the differential diagnosis of uterine tumors with prominent cystic degeneration in the clinical setting of relatively young women with abnormal uterine bleeding. 
ORCID: Ga Eun Park: http://orcid.org/0000-0002-2479-6950; Sung Eun Rha: http://orcid.org/0000-0003-1514-929X; Soon Nam Oh: http://orcid.org/0000-00032373-7024; Ahwon Lee: http://orcid.org/0000-0002-2523-9531; Keun Ho Lee: http://orcid.org/0000-0001-9005-7796; Mee-Ran Kim: http://orcid.org/0000-00034492-0768

\section{Conflict of Interest}

No potential conflict of interest relevant to this article was reported.

\section{Acknowledgments}

This study was supported in part by the Research Fund of the Korean Society of Ultrasound in Medicine.

\section{References}

1. D'Angelo E, Prat J. Uterine sarcomas: a review. Gynecol Oncol 2010;116:131-139.

2. Chan JK, Kawar NM, Shin JY, Osann K, Chen LM, Powell CB, et al. Endometrial stromal sarcoma: a population-based analysis. $\mathrm{Br} J$ Cancer 2008;99:1210-1215.

3. Gadducci A, Cosio S, Romanini A, Genazzani AR. The management of patients with uterine sarcoma: a debated clinical challenge. Crit Rev Oncol Hematol 2008;65:129-142.

4. Lin JF, Slomovitz BM. Uterine sarcoma 2008. Curr Oncol Rep 2008; 10:512-518.

5. Ueda M, Otsuka M, Hatakenaka M, Torii Y. Uterine endometrial stromal sarcoma located in uterine myometrium: MRI appearance. Eur Radiol 2000;10:780-782.

6. Rha SE, Byun JY, Jung SE, Lee SL, Cho SM, Hwang SS, et al. CT and MRI of uterine sarcomas and their mimickers. AJR Am J Roentgenol 2003;181:1369-1374.

7. Toprak U, Pasaoglu E, Karademir MA, Gulbay M. Sonographic, CT, and MRI findings of endometrial stromal sarcoma located in the myometrium and associated with peritoneal inclusion cyst. AJR Am J Roentgenol 2004;182:1531-1533.

8. Kim JA, Lee MS, Choi JS. Sonographic findings of uterine endometrial stromal sarcoma. Korean J Radiol 2006;7:281-286.

9. Fujii S, Kaneda S, Tsukamoto K, Kakite S, Kanasaki Y, Matsusue E, et al. Diffusion-weighted imaging of uterine endometrial stromal sarcoma: a report of 2 cases. J Comput Assist Tomogr 2010;34:377379.

10. Furukawa R, Akahane M, Yamada H, Kiryu S, Sato J, Komatsu S, et al. Endometrial stromal sarcoma located in the myometrium with a low-intensity rim on T2-weighted images: report of three cases and literature review. J Magn Reson Imaging 2010;31:975-979.

11. Conklin CM, Longacre TA. Endometrial stromal tumors: the new WHO classification. Adv Anat Pathol 2014;21:383-393.

12. Oliva $E$, Carcangiu ML, Carinelli SG, Ip P, Loening T, Longacre TA, et al. Mesenchymal tumors. In: Kurman RJ, Carcangui ML, Herrington CS, Young RH, eds. WHO classification of tumours of the female reproductive organs. 4th ed. Lyon: IARC Press, 2014;141-145.

13. Prat J. FIGO staging for uterine sarcomas. Int J Gynaecol Obstet 2009;104:177-178.

14. Perez-Montiel D, Salmeron AA, Dominguez Malagon H. Multicystic endometrial stromal sarcoma. Ann Diagn Pathol 2004;8:213-218.

15. Ali RA, El-Hady el SA, El-Zayat M. Multilocular intrauterine cystic mass, atypical presentation of endometrial stromal sarcoma: a case report. Arch Gynecol Obstet 2008;277:185-187.

16. Doghri R, Mrad K, Driss M, Sassi S, Abbes I, Dhouib R, et al. Endometrial stromal sarcoma presenting as a cystic abdominal mass. Pathologica 2009;101:93-96.

17. Jayakrishnan K, Koshy AK, Manjula P, Nair AM, Ramachandran A, Kattoor J. Endometrial stromal sarcoma mimicking a myoma. Fertil Steril 2009;92:1744-1746.

18. McCluggage WG, Cromie AJ, Bryson C, Traub Al. Uterine endometrial stromal sarcoma with smooth muscle and glandular differentiation. J Clin Pathol 2001;54:481-483.

19. Zaloudek C, Hendrickson MR. Mesenchymal tumors of the uterus. In: Kurman RJ, ed. Blaustein's pathology of the female genital tract. 5th ed. New York: Springer, 2002;561-616.

20. Ali RH, Rouzbahman M. Endometrial stromal tumours revisited: an update based on the 2014 WHO classification. J Clin Pathol 2015;68:325-332

21. Cho FN, Liu CB, Yu KJ. Low-grade endometrial stromal sarcoma initially manifesting as a large complex pedunculated mass arising from the uterine surface. Ultrasound Obstet Gynecol 2011;38:233234.

22. Somma A, Falleti J, Di Simone D, Marra A, Nazzaro G, Miranda M, et al. Cystic variant of endometrial stromal sarcoma: report of two cases. Int J Surg Pathol 2013;21:278-281.

23. Wood CL, Sederberg J 2nd, Russ P, Seres T. Cystic appearance of low-grade endometrial stromal sarcoma in the right atrium: case report. Cardiovasc Ultrasound 2011;9:23.

24. Itoh T, Mochizuki M, Kumazaki S, Ishihara T, Fukayama M. Cystic pulmonary metastases of endometrial stromal sarcoma of the uterus, mimicking lymphangiomyomatosis: a case report with immunohistochemistry of HMB45. Pathol Int 1997;47:725-729. 